\title{
RapidAIM: a culture- and metaproteomics- based Rapid Assay of Individual Microbiome responses to drugs
}

\author{
Leyuan $\mathrm{Li}^{1}$, Zhibin Ning ${ }^{1}$, Xu Zhang ${ }^{1}$, Janice Mayne ${ }^{1}$, Kai Cheng ${ }^{1}$, Alain Stintzi ${ }^{{ }^{*}}$ and Daniel Figeys ${ }^{1,2^{*}}$ (D)
}

\begin{abstract}
Background: Human-targeted drugs may exert off-target effects or can be repurposed to modulate the gut microbiota. However, our understanding of such effects is limited due to a lack of rapid and scalable assay to comprehensively assess microbiome responses to drugs. Drugs and other compounds can drastically change the overall abundance, taxonomic composition, and functions of a gut microbiome.

Results: Here, we developed an approach to screen compounds against individual microbiomes in vitro, using metaproteomics to both measure absolute bacterial abundances and to functionally profile the microbiome. Our approach was evaluated by testing 43 compounds (including 4 antibiotics) against 5 individual microbiomes. The method generated technically highly reproducible readouts, including changes of overall microbiome abundance, microbiome composition, and functional pathways. Results show that besides the antibiotics, the compounds berberine and ibuprofen inhibited the accumulation of biomass during in vitro growth of the microbiota. By comparing genus and species level-biomass contributions, selective antibacterial-like activities were found with 35 of the 39 non-antibiotic compounds. Seven of the compounds led to a global alteration of the metaproteome, with apparent compound-specific patterns of functional responses. The taxonomic distributions of altered proteins varied among drugs, i.e., different drugs affect functions of different members of the microbiome. We also showed that bacterial function can shift in response to drugs without a change in the abundance of the bacteria.
\end{abstract}

Conclusions: Current drug-microbiome interaction studies largely focus on relative microbiome composition and microbial drug metabolism. In contrast, our workflow enables multiple insights into microbiome absolute abundance and functional responses to drugs. The workflow is robust, reproducible, and quantitative and is scalable for personalized high-throughput drug screening applications.

Keywords: Gut microbiome, Drug response, In vitro culturing, Metaproteomics, Absolute abundance, Functional profile

\section{Background}

Human-targeted drugs are primarily developed for their effects on the host, and little is known on their effects on the microbiome. Microbiome response to drugs could contribute to off-target drug effect [1]. In addition, the gut microbiome has been linked to gastroenterological, neurologic, respiratory, metabolic, hepatic, and cardiovascular diseases [2]. Therefore, targeting the microbiome could lead to novel therapies [3]. Orally administrated drugs go

\footnotetext{
* Correspondence: astintzi@uottawa.ca; dfigeys@uottawa.ca

'Department of Biochemistry, Microbiology and Immunology, Ottawa Institute of Systems Biology, Faculty of Medicine, University of Ottawa, Ottawa, Canada

Full list of author information is available at the end of the article
}

through complex processing by the host [4], with some drugs reaching the microbiome. As well, drugs can be repurposed to target the microbiome using enteric/colonic delivery approaches [5]. Although the effects of some drugs and compounds on the microbiome have been reported [6], many drug-microbiome interactions are unknown. This is due in part to the extremely high numbers of marketed drugs [7] and compounds in development [8] together with the lack of assays that can rapidly and comprehensively assess the effects of compounds on individual microbiomes.

Different in vitro approaches have been employed to study drug-microbiome interactions. One strategy involves long-term stabilization of the microbiota, as shown in 
various intestinal microbiota simulators based on continuous flow [9-11]. This approach typically requires a long culture period to stabilize the microbiota (15-20 days), and notable shifts in taxonomic compositions compared with the inoculum have been shown $[9,12]$. Moreover, the size and complexity of these culturing systems limit the number of individual microbiomes and drugs that can be examined [11], and thus may not be suitable for high-throughput drug screening purpose. Another strategy is to culture individual bacteria strains isolated from microbiomes. A recent study examined the effects of approved drugs on the biomass of forty individually cultured bacterial strains in a high-throughput manner [13]. This approach highlighted the importance of biomass in identifying antibacterial effects. However, it did not take into account the complexity of a microbial community that could lead to different microbial responses. Approaches such as optical density measurement [13], flow cytometry [14], and quantitative realtime PCR [15] can be used to compare microbiome biomass. However, these approaches lack insights into drug impacts on microbial composition and functions, which are highly related to healthy and disease states. There has been no report of an in vitro gut microbiome-based drug screening approach that could assess both biomass responses and functional alterations in a single analytical test.

The development of meta-omics approaches allowed rapid and deep measurement of microbiome compositions and functional activities. Genetic approaches such as sequencing of $16 \mathrm{~S}$ rRNA gene fragment amplicons and shotgun metagenomics have been regarded as the "gold standard" in microbiome analysis, providing relative quantifications of microbiome composition and functional capabilities [16, 17]. Notably, different microbial members can differ by several orders of magnitude in biomass [18]. Moreover, there is little insight on which microbial traits actually contribute to the functional activities of the microbiome, as functions predicted from metagenomics analyses are not necessarily expressed. Studies have shown that gene copy numbers are not representative of protein levels [19]. In addition, RNA expression have limited correlation to the actual protein abundance [20]. In contrast, mass spectrometry (MS)-based metaproteomics technology allows for deep insight into proteome-level information of the microbiome $[21,22]$, providing quantified protein abundances that estimate the functional activities of the microbiome. Proteins not only provide the biological activities to the microbiome but also contribute the majority of biomass in microbial cells. Hence, the metaproteomic readouts can also be used to assess the microbiome biomass and analyze community structure [23]. Metaproteomics has been previously validated to estimate the microbiome and individual microbe biomasses
[24], and it readily quantifies the bacterial species responsible for $>90 \%$ of the total microbiome biomass [25], making it sufficient for a fast-pass drug screening application.

Here, we report an approach named Rapid Assay of Individual Microbiome (RapidAIM), which applies metaproteomics to gain insights into the microbiome responses to drugs in an in vitro model [26]. Forty-three compounds that have been previously suggested to impact, interact with, or be metabolized by the gut microbiome were selected for this study (Supplementary Table S1). Briefly, in RapidAIM, individual microbiomes are cultured in a previously optimized culture system [26] for $24 \mathrm{~h}$, and the samples are then analyzed using a metaproteomics-based analytical approach. A highthroughput equal-volume based protein extraction and digestion workflow was applied to enable absolute biomass assessment along with the functional profiling. To demonstrate the feasibility and performance of the RapidAIM assay, we carried out a proof-of-concept study involving the 43 compounds and 5 individual gut microbiomes. Microbiome responses including changes in biomass, taxon-specific biomass contributions, taxonspecific functional activities, and detailed responses of specific enzymatic pathways can be obtained following the assay.

\section{Results}

\section{Development and evaluation of RapidAIM}

RapidAIM consists of an optimized microbiome culturing method, an equal-volume based protein extraction and digestion workflow and a metaproteomic analysis pipeline (Fig. 1a). Briefly, fresh human stool samples are inoculated in 96-well deep-well plates and cultured with drugs for $24 \mathrm{~h}$. We have previously optimized the culture model and validated that it maintains the composition and taxon-specific functional activities of individual gut microbiomes in 96-well plates [26]. After 24 $\mathrm{h}$, the cultured microbiomes are prepared for metaproteomic analysis using a microplate-based metaproteomic sample processing workflow (Supplementary Figure S1) adapted from our single-tube protocol [28]. The microplate-based workflow consists of bacterial cell purification, cell lysis with ultrasonication in $8 \mathrm{M}$ urea buffer, in-solution tryptic digestion, and desalting. We validated each step of this workflow and found no significant differences in identification efficiency between 96-well plate processing and single-tube processing (Supplementary Figure S1). To compare total biomass, taxon-specific biomass and pathway contributions between samples in a high-throughput assay format, we applied an equal sample volume strategy to our recently developed metaproteomics techniques [22, 27, 29]. To validate the absolute quantification of microbiome 


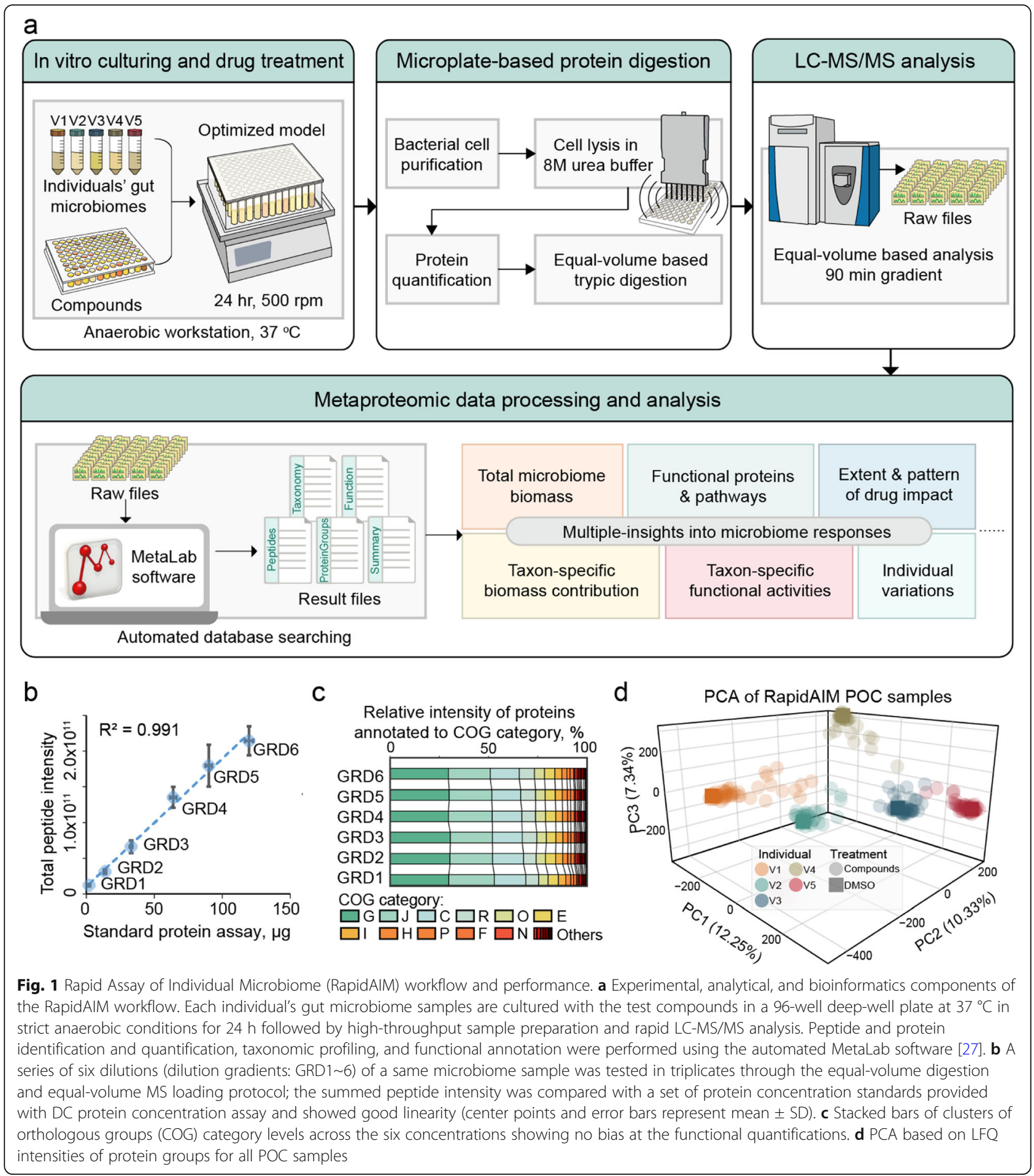

abundance by comparing total peptide intensity, an equal volume of samples from a microbiome dilution series (simulating different levels >of drug effects) was taken for tryptic digestion and LC-MS/MS analysis. Summed peptide intensity in each sample showed good linearity $\left(R^{2}=0.991\right.$, Fig. $\left.1 \mathrm{~b}\right)$ with a standard colorimetric protein assay, showing that the total peptide intensity is a good indicator for microbiome biomass levels. Because drugs can cause drastic changes in microbiome abundance, we then evaluated whether biomass differences between wells could also cause bias in identified functional and taxonomic compositions. We confirmed that the level of total biomass did not bias the composition of functional profiles (Fig. 1c), 
protein groups (Supplementary Figure S2a), or taxonomic abundances (Supplementary Figure S2b).

\section{RapidAIM: proof-of-concept study}

We conducted a proof-of-concept (POC) study on the use of RapidAIM to characterize drug effect on the microbiome. We selected 43 compounds that have been previously suggested to impact, interact with, or be metabolized by the gut microbiome (Supplementary Table S1). Thirty-seven of these compounds are FDAapproved drugs; 4 are antibiotics, and the others include nonsteroidal anti-inflammatory drugs (NSAIDs), antidiabetic drugs, aminosalicylate, and statins. Each compound, at a concentration corresponding to the maximal daily oral dose distributed in 200 g daily fecal wet weight [30], was added to 5 wells of 96-well plates containing 1$\mathrm{mL}$ culture medium in each well. The drug solvent, dimethyl sulfoxide (DMSO), was used as the negative control. Then, each of the 5 wells for each compound was inoculated with a different fecal microbiome from healthy human volunteers. Following $24 \mathrm{~h}$ of culturing, the samples were processed through the microplatebased workflow (Supplementary Figure S1) and were subjected to a 90 min gradient-based rapid LC-MS/MS analysis. Using our automated metaproteomic data analysis software MetaLab [27], 101,995 peptide sequences corresponding to 24,631 protein groups were quantified across all samples with a false discovery rate (FDR) threshold of 1\% (Supplementary Figure S3a). The average MS/MS identification rate was $32.4 \pm 8.8 \%$ (mean \pm $\mathrm{SD})$; an average of $15,017 \pm 3654$ unique peptides and $6684 \pm 998$ protein groups were identified per sample (Supplementary Figure S3c-d). To provide a global overview of the microbiome responses, a PCA was performed based on label-free quantification (LFQ) intensities of protein groups (Fig. 1d). As expected, the samples clustered based on the original microbiome source and not based on drug treatment. A PerMANOVA test [31] based on Bray-Curtis dissimilarities [32, 33] showed that the samples were significantly clustered according to different individuals $(p=0.001$ based on 999 permutations). Within each individual microbiome group, a number of drug-treated samples clustered closely to their control, while several other samples clearly separated from the non-treated control.

We next evaluated the robustness and reproducibility of the method by culturing one microbiota with drugs in technical triplicates. Cultured triplicates yielded high Pearson's $r$ for LFQ protein group intensities (Supplementary Figure S3b). Hierarchical clustering based on Pearson's $r$ of LFQ protein group intensities between samples showed that with the exception of several compounds which clustered closely with DMSO; cultured triplicates were clustered together (Supplementary Figure S4a). Moreover, total biomass, functional enzymes, and species biomass contributions were highly reproducible between triplicates, as shown in Supplementary Figure S4b-d.

\section{Effects of compounds on microbiome abundance and composition}

We examined the effect of the 43 compounds on the overall abundance (biomass) of each individual microbiome by comparing the total peptide intensity (Fig. 2a). As expected, the antibiotics greatly reduced total microbial biomass in most individual microbiomes (with one exception of increased microbiome abundance in response to rifaximin, further examination is shown in Supplementary Figure S5). Similar to these antibiotics, berberine and ibuprofen also inhibited the biomass of all individual microbiomes.

We next explored the effects of drugs on the microbiome composition based on bacterial biomass contributions. To evaluate the overall shift of the microbiome, Bray-Curtis distance $[32,33]$ between drug-treated and DMSO control microbiome indicated that fructooligosaccharide (FOS), rifaximin, berberine, diclofenac, ciprofloxacin, metronidazole, and isoniazid significantly shifted the microbiome (pairwise Wilcoxon test, FDR-adjusted $p<0.05$; Fig. 2c).

We further examined the response of individual bacterial taxon using their absolute abundance estimated using the sum of all peptide intensities for each taxon as previously described [27] (Fig. 2b). In contrast to genetic sequencing-based approaches, which often only report relative abundance, metaproteomics measure absolute abundances. As expected, the broad-spectrum antibiotics rifaximin, ciprofloxacin, and metronidazole significantly reduced the total biomass (Fig. 2a) and the absolute abundance of a many bacterial genera (Fig. 2b, Wilcoxon test, $p<0.05$ ). Nevertheless, ciprofloxacin and metronidazole significantly increased the relative abundance of genera Bifidobacterium, Ruminococcus, Butyrivibrio, Paenibacillus, etc. (Supplementary Figure S6). Nonantibiotic compounds, such as berberine, FOS, pravastatin, ibuprofen, diclofenac, flucytosine, and indomethacin also showed significant decreases in the abundances of over 10 genera. In addition, selective antibacterial activities were found in 35 out of the 39 non-antibiotic compounds at the genus level; at the species level, we found that 32 non-antibiotic compounds significantly altered the biomass of at least one bacterial species (onesided Wilcoxon rank sum test, FDR-adjusted $p<0.05$; Supplementary Table S2). Interestingly, members of the Actinobacteria phyla, including Eggerthella, Gordonibacter, Slackia, and Adlercreutzia, were the most susceptible to drugs compared with most other genera (Supplementary Figure S6). In summary, RapidAIM allowed for the assessment of changes in both absolute and relative abundances of microbes in response to compounds. 


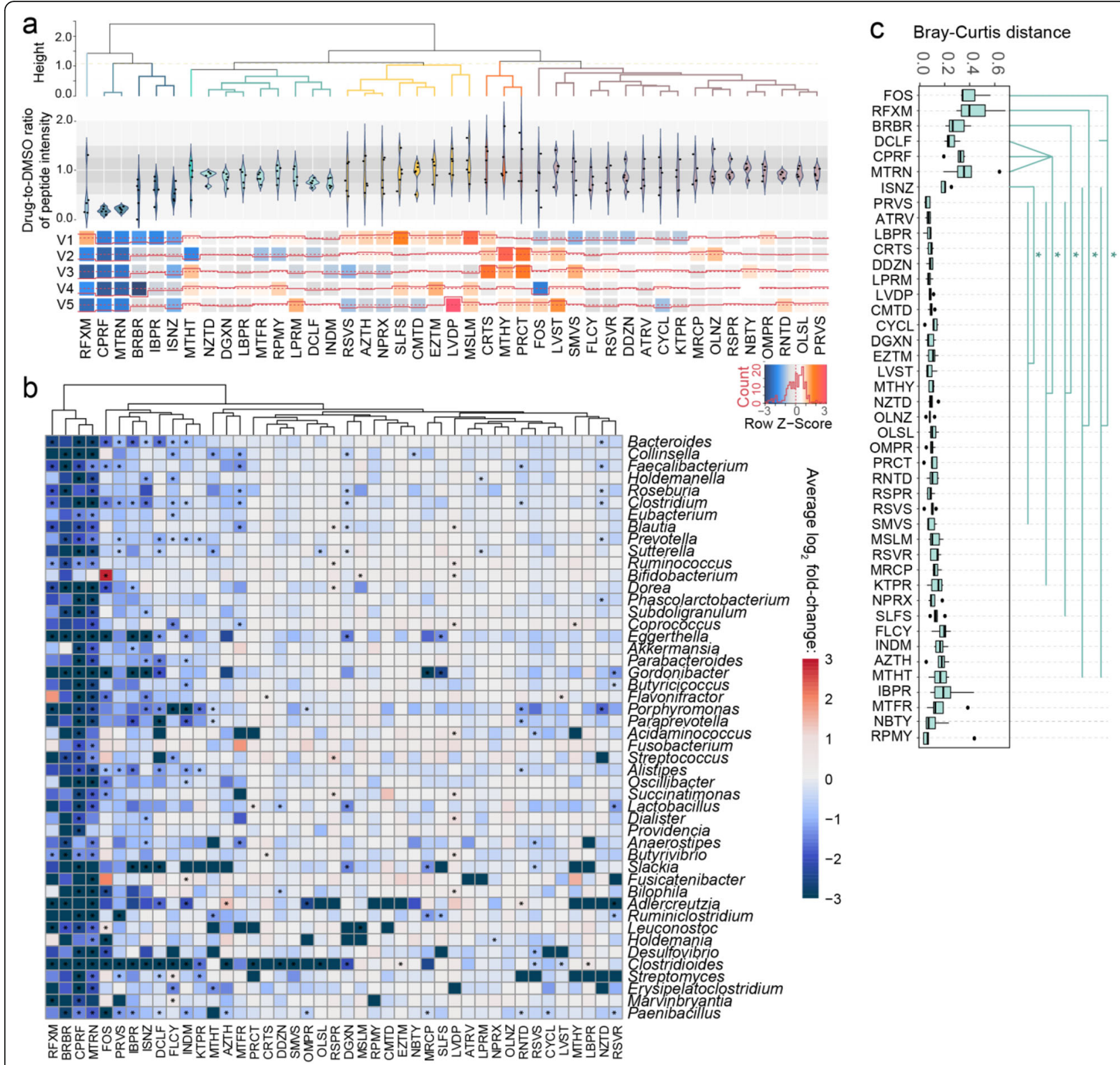

Fig. 2 Response of microbiome abundance and composition to compounds. a Biomass responses of individuals' microbiomes to compounds relative to DMSO control. Ratio of peptide intensity between compound and DMSO control samples was calculated for each individual microbiome. $\mathbf{b} \log _{2}$ fold-change of absolute abundance at the genus level in response to each drug compared with the DMSO control. Genera that existed in $\geq 80 \%$ of the volunteers are shown. Asterisk indicates significantly changed bacterial abundance by Wilcoxon test, $p<0.05$. $\mathbf{c}$ BrayCurtis distance of genus-level composition between drug-treated microbiomes and the corresponding DMSO control samples. Heatmap colors are generated with average of $\log _{2}$ fold-changes among the five individual samples. Statistical significance was calculated by pairwise Wilcoxon test (FDR-adjusted $p<0.05$ ). Box spans interquartile range (25th to 75th percentile), and line within box denotes median. For full compound names, see abbreviation list in Supplementary Table S1

\section{Overall functional profiles in response to compounds}

The Bray-Curtis distance of protein group profiles showed that all the four antibiotics, as well as FOS, berberine, and diclofenac, significantly altered the microbiome functions (Fig. 3a). These functional alterations likely stemmed from changes in taxonomic composition as revealed by the genus-level Bray-Curtis distance analysis (Fig. 2c). We next analyzed the protein group intensities by partial least square discriminant analysis (PLS-DA) to determine whether metaproteomic profiles could be used to discriminate between the DMSO control and each of the drug-treated microbiomes. In agreement with the Bray-Curtis analysis results, PLS-DA interpretation identified drug-specific metaproteomic 

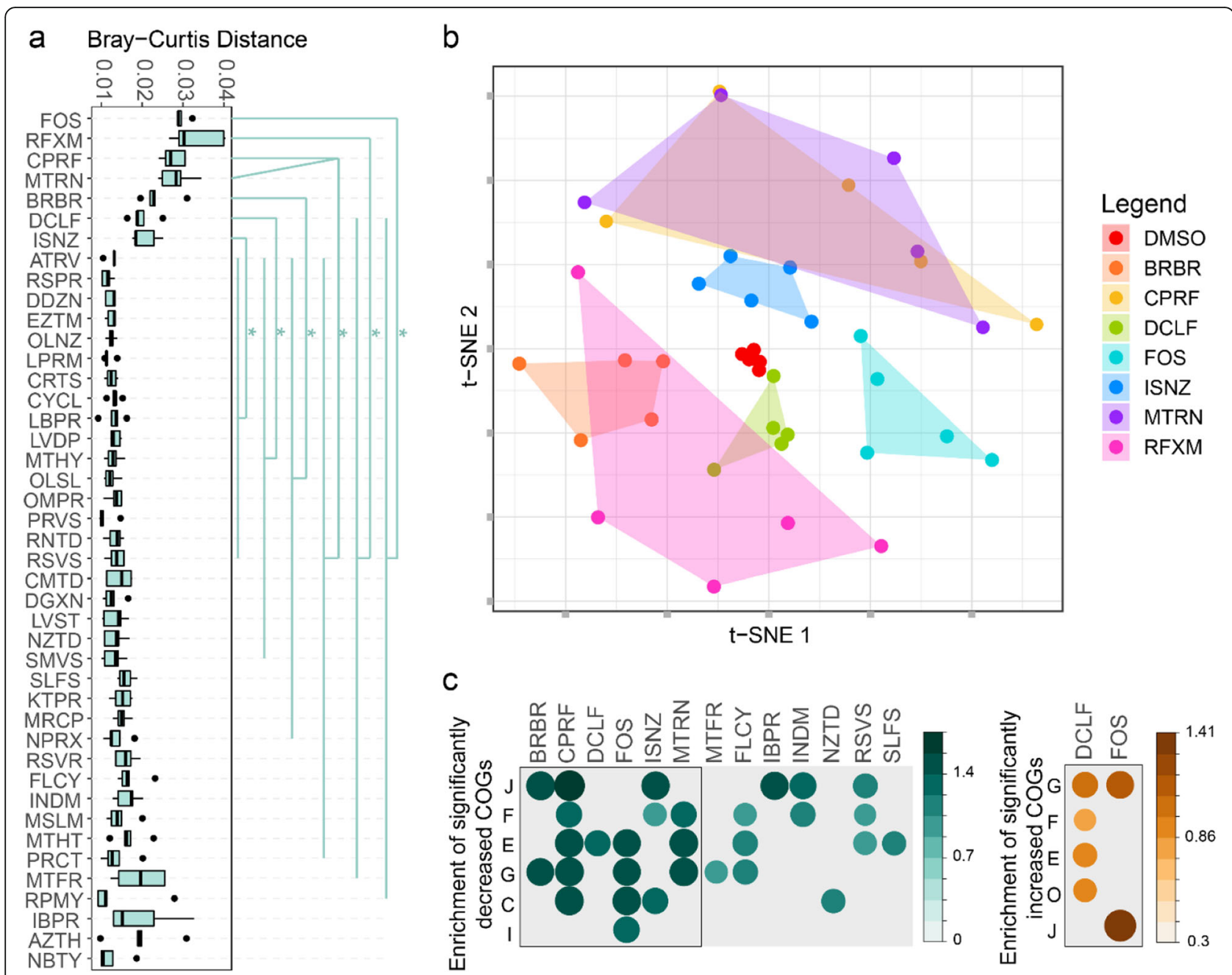

Fig. 3 Effect of compounds on metaproteomic profiles of the microbiome. a Bray-Curtis distance of protein groups between drug-treated microbiomes and the corresponding DMSO control samples. Statistical significance was calculated by pairwise Wilcoxon test (FDR-adjusted $p<$ 0.05). b Unsupervised dimensionality reduction analysis showing compound-specific patterns of responses to class I compounds. c Enrichment analysis of all significantly different COGs in the POC dataset. Significantly altered COGs with a $p$ value cutoff of 0.05 are shown

patterns associated with the seven abovementioned compounds, i.e., the four antibiotics, FOS, berberine, and diclofenac (Supplementary Figure S7). Hence, hereafter, we named these seven compounds as class I compounds, whereas others were named class II compounds. To gain a better understanding of the global effects of class I compounds on the gut microbiome, we applied an unsupervised non-linear dimensionality reduction algorithm, t-distributed stochastic neighbor embedding ( $t-\mathrm{SNE}$ ) [34], to visualize this subgroup of metaproteomic data based on protein group abundances (Fig. 3b). Class I compounds led to a global alteration of the metaproteome, with apparent compound-specific patterns.

We next examined the drug impacts on the abundance of functional proteins according to clusters of orthologous groups (COG) of proteins. We identified 535 COGs significantly decreased by at least 1 drug treatment; 15 of these COGs were decreased by $\geq 10$ compounds (Supplementary Figure S8). Diclofenac and FOS were the only 2 compounds that significantly increased COGs (55 and 81 COGs, respectively). Enrichment analysis based on these significantly altered COGs shows that COG categories found to be enriched were responsive to 13 of our compounds (Fig. 3c), 6 of those were class I compounds. Interestingly, the nonantibiotic NSAID diclofenac increased the abundance of several COG categories (Fig. 3c). By mapping these significantly increased proteins from these COG categories against the string database, we found that these altered proteins are functionally interconnected (Supplementary Figure S9). 


\section{Specific proteins and enzymatic pathways in response to compounds}

Next, we examined the ability of RapidAIM to observe the modulations of specific proteins and enzymatic pathways of interest. As an example, we show that proteins related to drug resistance were significantly altered by several compounds (Wilcoxon test, Fig. 4a). Multidrug efflux pump proteins that could extrude structurally dissimilar organic compounds [35] were significantly increased by ibuprofen and sulfasalazine treatment. Antibiotic-degrading enzyme beta-lactamase [36] was significantly decreased by the antibiotics ciprofloxacin and metronidazole. Thioredoxin participates in defense against oxidative stress induced by drugs via peroxiredoxin [37]. Significant increases in thioredoxin and/or peroxiredoxin were observed in ten of our treatments. In addition, we analyzed the response of enzymes along the butyrate production pathway. In response to the antifungal drug flucytosine, five out of seven observed enzymes showed significant decreases (Supplementary Figure S10). As another group of examples, we show the effects of FOS and ciprofloxacin on specific enzymatic pathways in an individual microbiome view (Fig. 4b, c). Protein groups were annotated to KEGG (Kyoto Encyclopedia of Genes and Genomes) enzymes and were mapped against the KEGG pathway database. FOS increased enzymes responsible for fructan and sucrose uptake, as well as enzymes for conversion of D-fructose into

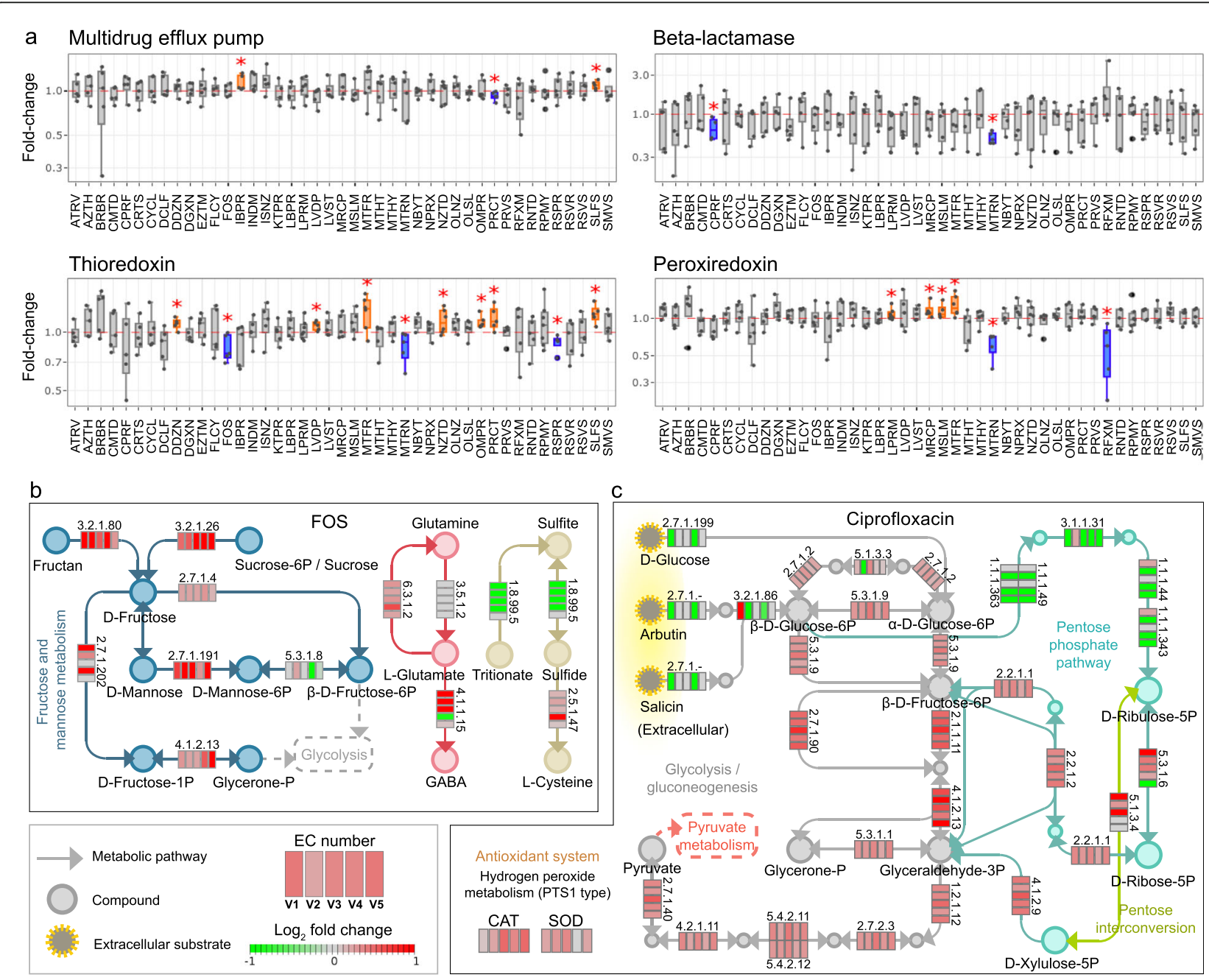

Fig. 4 Response of specific proteins and enzymatic pathways to drug treatment. a Response of proteins related to drug resistance (red asterisk denotes $p<0.05$ by Wilcoxon test); boxplots of all functional responses of the RapidAIM POC dataset are available at https://shiny.imetalab.ca/ RapidAIM_functional_response/. b Effect of FOS treatment on enzymes involved in fructose and mannose metabolism, GABA production, and sulfide metabolism pathways. c Effect of ciprofloxacin treatment on enzymes involved in the glycolysis/gluconeogenesis and pentose phosphate pathway. The five blocks of each enzyme represent the five individual microbiomes. Colors in the blocks represent differences between normalized KEGG enzyme intensities with drug treatment versus DMSO $\left(\log _{2}\right.$ transformation of the original intensity followed by a quotient normalization $(x /$ mean)) 
D-fructose-1-phosphate, D-mannose-6-phosephate, and $\beta$-D-fructose-6-phosphate (Fig. 4b). FOS also affected enzymes involved in the interconversion between glutamine, glutamate, and GABA (molecules involved in gut-brain communication). In addition, enzymes involved in sulfide accumulation were affected, including decrease of dissimilatory sulfite reductase (EC 1.8.99.5) and increase of cysteine synthase (EC 2.5.1.47) (Fig. 4b). Ciprofloxacin significantly altered the levels of enzymes involved in glycolysis/glycogenesis and pentose phosphate pathways (Fig. 4c). The majority of enzymes involved in glycolysis were significantly increased by ciprofloxacin. Ciprofloxacin down-regulated enzymes (ECs 1.1.1.49/1.1.1.363, 3.1.1.31, 1.1.1.44/1.1.1.343) involved in synthesis of ribulose-5-phosphate, which can be isomerized to ribose 5phosphate for nucleotide biosynthesis [38]. Moreover, the levels of antioxidant enzymes superoxide dismutase (SOD) and catalase (CAT) were increased, suggesting that ciprofloxacin induces oxidative stress in gut bacteria (Fig. 4c).

Taxon-specific functional responses to class I compounds We next performed a taxonomic analysis correlating to the functional responses to diclofenac, FOS, ciprofloxacin, and berberine, which represent four different types of compounds (NSAID, oligosaccharide, antibiotics, anti-diabetes) in the class I. Protein groups with VIP scores $>1$ (thereafter defined as differential proteins) were extracted from each model and were annotated with their taxonomic and COG information. The taxonomic distributions of the differential proteins varied among drugs (Fig. 5a). Moreover, mapping of the differential proteins to phyla-specific pathways revealed phyla-specific responses, as shown for berberine in Supplementary Figure S11. In agreement with Fig. 5a, proteins with decreased abundance had a higher pathway coverage than the proteins increased in Firmicutes and Actinobacteria, while the opposite pattern was observed in Bacteroidetes, Proteobacteria, and Verrucomicrobia. In some cases, the phylum-specific responses included both increased and decreased proteins within the same pathway (black lines, Supplementary Figure S11). For example, we observed this pattern in fatty acid, carbohydrate, and nucleotide metabolism pathways in Firmicutes.

Genus-level analysis revealed genus-specific responses to berberine (Fig. 5b). In most genera, the genus-specific responses correlated with the overall abundance of the corresponding genus (Fig. 5b, right panel). Nevertheless, some genera showed functional shifts in response to berberine without changes in overall abundance. For example, Bifidobacteria, Roseburia, Eubacterium, Clostridium, Ruminococcus, Blautia, and Subdoligranulum exhibited downregulation of proteins in various COG categories but no changes in biomass were observed.
Gut microbiome functions altered by class II compounds Class II compounds, in contrast to class I compounds, did not cause a global shift in the five individual microbiomes (an example is given by indomethacin, Fig. 6a). However, Fig. 6a as well as the Bray-Curtis analyses (Figs. 2c and 3a) suggested that there was individual variability in the extent of drug response. We show that when analyzed on an individual subject basis, significant individualized functional effects are present. For example, PCA showed a clear differentiation between indomethacin-treated microbiome and the DMSO control in cultured replicates of a single indomethacintreated microbiota from individual V1 (Fig. 6b), where, 303 significantly altered protein groups were identified (Fig. 6c). These protein expression shifts suggest high sensitivity of the RapidAIM assay in its application to personalized drug screenings. Taxon-function coupled enrichment analysis showed that protein groups with decreased abundances were highly enriched in the genus Bacteroides. In addition, increased protein groups were also enriched in Gordonibacter pamelaeae, Firmicutes bacterium CAG:102, Alistipes putredinis, Eggerthella genus, etc. (Fig. 6d). Protein groups with increased abundances were mostly enriched in the order Enterobacterales and were also enriched in Burkholderiales order, Collinsella genus, and Proteobacteria phylum (Fig. 6d, e). We further analyzed the functional enrichment corresponding to the increased protein groups. Most of the enriched functions corresponded to Enterobacterales. These enriched functions included molecular chaperones COG0459 chaperonin GroEL (HSP60 family) and COG0234 co-chaperonin GroES (HSP10) (Fig. 6e).

\section{Discussion}

In the present study, we developed an approach named RapidAIM to evaluate the effects of xenobiotics on individual microbiomes. The range of xenobiotics that reach the intestine and may interact with the gut microbiome is massive and expanding. These xenobiotics include antibiotics and other pharmaceuticals, phytochemicals, polysaccharides, food additives, and many other compounds. With the exception of antibiotics, we remain surprisingly ignorant on the extent to which these compounds affect the functions of the gut microbiome, and whether these compounds could be repurposed to provide beneficial effects. This understanding was limited by the lack of an efficient and scalable approach that could maximally obtain insights into microbiome responses while minimizing the number of analytical tools being used.

Here, we describe an approach which enables the exploration of drug-microbiome interactions using an optimized in vitro culturing model and a metaproteomic approach. We have achieved the maintenance of the 


\begin{tabular}{|c|c|c|c|}
\hline a & Berberine & Ciprofloxacin & \\
\hline Firmicutes & Poe & 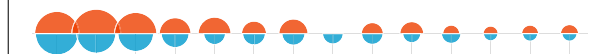 & $\begin{array}{l}\text { Size } \\
\qquad d=n^{1 / 3}\end{array}$ \\
\hline Bacteroidetes & 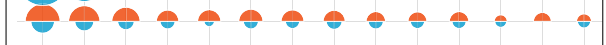 & 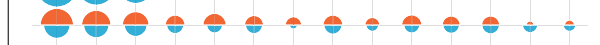 & \\
\hline Proteobacteria & 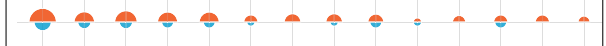 & 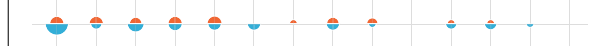 & \\
\hline Actinobacteria & 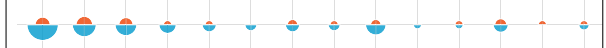 & $\theta=\theta=$ & \\
\hline Verrucomicrobia & $a-\infty \ldots, \ldots, \ldots$ & $=$. & $150 \frac{\bar{c}}{\mathbb{0}}$ \\
\hline & $\begin{array}{lllllllllllllll} & \text { J } & \text { C } & E & O & R & P & M & F & \text { I } & H & K & L & T \\
\text { FOS } & & & & & & & & & & & & & & \\
\end{array}$ & $\begin{array}{llllllllllll} & \mathrm{J} \\
\text { Diclofenac } & & & & & & & & & & & \end{array}$ & 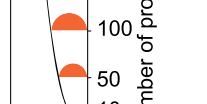 \\
\hline Firmicutes & $\theta \bullet \theta \bullet \bullet \bullet$ & 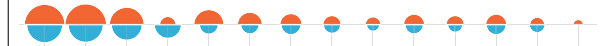 & $H_{1}^{10} \bar{z}$ \\
\hline Bacteroidetes & 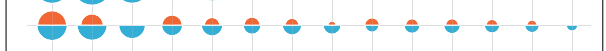 & 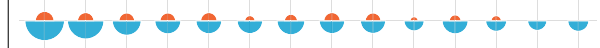 & Regulation \\
\hline Proteobacteria & $\in=0=0=0$ & 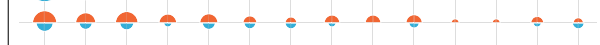 & Upregulated \\
\hline Actinobacteria & 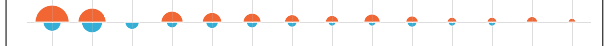 & 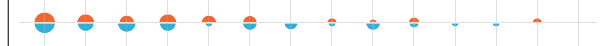 & Downregualted \\
\hline Verrucomicrobia & 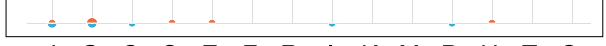 & $-\ldots, \ldots \quad=1$ & \\
\hline
\end{tabular}

b

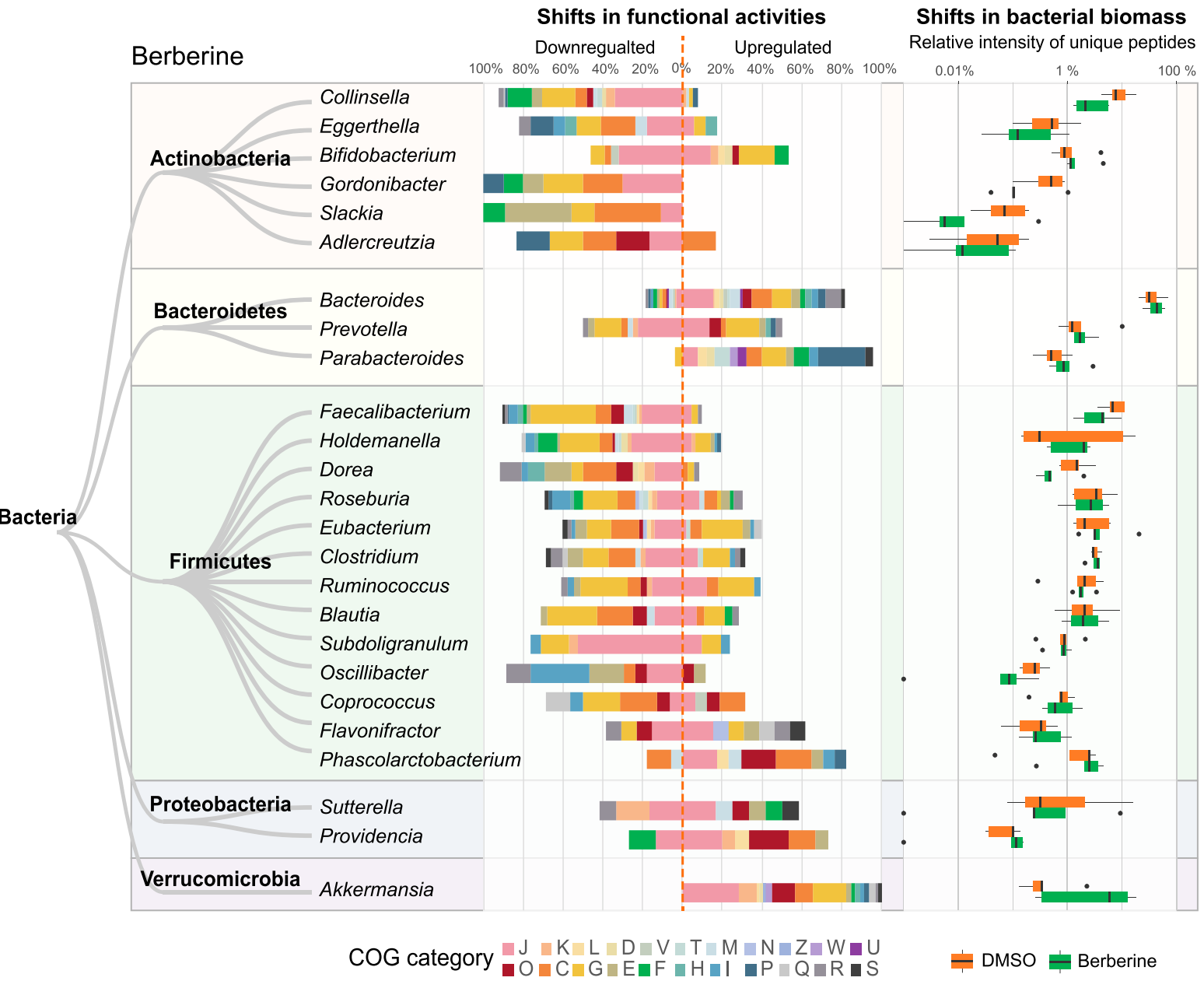

Fig. 5 Global functional effects of berberine, ciprofloxacin, FOS and diclofenac. a Taxon-function distribution of protein groups responding to berberine, FOS, ciprofloxacin, and diclofenac. Responding protein groups were selected by PLS-DA based on ComBat-corrected data. The semicircle diameter represents the number of PLS-DA VIP $>1$ protein groups corresponding to each phyla-COG category pair. b Genus level shifts in functional activities in response to berberine and the alterations in biomass of the corresponding genera. Functional shifts (differential protein groups) were identified by PLS-DA. For each genus, the percentages of the total numbers of up- and downregulated protein groups corresponding to each COG category are shown. Shifts in bacterial biomass in the five microbiomes are shown in box plots with the boxes spanning interquartile range (25th to 75 th percentile) and the vertical lines denoting the median for each genus 


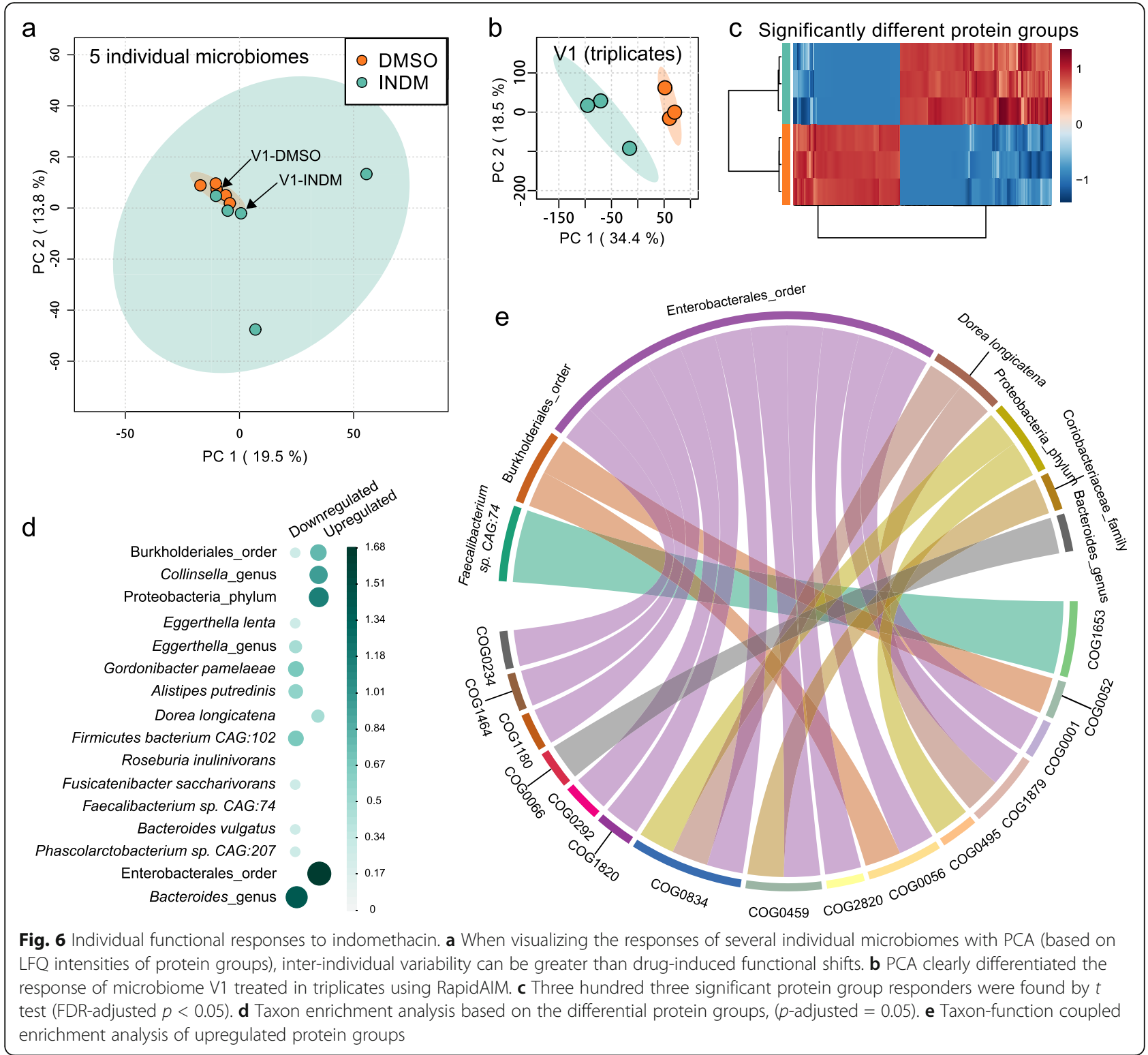

representativeness of the initial individual microbiome [26]. Furthermore, for an in vitro culturing simulating the in vivo microbiome, it is important to note that the population of gut bacteria in the human body is highly dynamic. It has been estimated that there are $\sim 0.9 \cdot 10^{11}$ bacteria/g wet stool and a total of $\sim 3.8 \cdot 10^{13}$ bacteria in the colon. Approximately 200-g wet daily stool would be excreted [30], leading to a dramatic decrease of the bacterial number in the gut; on the other hand, new bacterial biomass starts growing on nutrients passing through the gut. Current technologies examining the effect of xenobiotic stimulation are usually based on microbiota stabilized after over 2 weeks of culturing. However, at the stable phase of microbiota growth, the ecosystem reaches its carrying capacity (stable population size), limiting possible observations such as drug effect on the biomass. We have previously validated that the composition of gut microbiota is well maintained along the growth curve using our growth cultures [26], and we were able to observe drug responses of growing gut microbiota by adding the compounds at the initial inoculation stage. Subsequently, combined with our quantitative metaproteomics approach based on equal-sample volume digestion, we were able to observe the drug responses of using the overall microbiome abundance and taxon-specific biomass contributions.

Absolute abundance is a much better measurement of drug effects on the microbiome compared with relative abundance because it details the levels of individual bacteria as well as the summative response at the 
microbiome level. Moreover, it allows comparisons of effects across multiple drugs. Nevertheless, as the metagenomics field predominantly uses techniques that only report relative abundance, we also provided the relative abundance in Supplementary Figure S6. At first glance, relative and absolute abundance can appear to tell different stories. For example, Bifidobacterium is known to be resistant to ciprofloxacin and metronidazole [39] whereas most other genera are not. Therefore, while the total biomass was decreased by ciprofloxacin and metronidazole, the absolute abundance of Bifidobacterium remained the same, resulting in an overall increase in its relative abundance. Ibuprofen has been frequently used as a safe medication; however, a recent study suggested that ibuprofen had antimicrobial activity as a side effect [40]. In agreement, we showed that ibuprofen significantly inhibited the overall microbiome biomass, involving decreased absolute abundances of common gut commensals such as Bacteroides, Clostridium, Dorea, Eggerthella, and Akkermansia. As a final example, previous studies suggested that berberine has positive effect on beneficial gut microbes, e.g., selectively enriched a few putative short-chain fatty acid producing bacteria [41], and increased the relative abundance of Akkermansia [42]. However, although we also observed an increase in Akkermansia's relative abundance, its absolute abundance was not affected by berberine. In this example, an enrichment of a taxon (increase in relative abundance) does not necessarily relate to its outgrowth. Therefore, we suggest that absolute abundance provides a better evaluation of the changes induced in the microbiome by drug treatments.

We showed that the RapidAIM assay yielded insights into functional responses at multiple levels. Using PLSDA, we found that berberine, FOS, metronidazole, isoniazid, ciprofloxacin, diclofenac, and rifaximin consistently shifted the metaproteome of the individual gut microbiomes. By annotating the altered proteins at taxonomy, function, and pathway levels, we revealed the actions of the different drugs on the microbiome. For example, FOS treatment elevated enzymes involved in fructan and sucrose uptake, as well as enzymes involved in the interconversion among glutamine, glutamate, and GABA, which are associated with microbiome communication via the gut-brain axis [43]. In agreement, a study has shown that FOS administration increased GABA receptor genes in mice and further exhibited both antidepressant and anxiolytic effects [44]. FOS also decreased proteins involved in sulfide generation, suggesting decreased sulfide accumulation in the microbiome. This observation is in agreement with in vivo studies showing that FOS treatment decreased the concentration of fecal $\mathrm{H}_{2} \mathrm{~S}$ [45-47]. Ciprofloxacin treatment increased enzymes SOD and CAT, which was in agreement with several reports indicating that ciprofloxacin triggers oxidative stress in several bacteria [48-50]. With berberine treatment, we showed that taxonspecific functional shifts can occur either with or without a change in the taxon's biomass. Our analysis on phylum-specific responses showed that within the same phylum, proteins with the same functionality can increase in one species while decreasing in another, suggesting that there can be strong functional redundancy of species within a phylum. These observations highlight the strength of our workflow which enables quantitative metaproteomic profiling of the microbiome. Indeed, current classical sequencing-based approaches (sequencing of 16S rRNA gene fragment amplicons or metagenomics sequencing), which generate relative abundances, would not detect these types of changes. Finally, we showed that although a compound may not show global impacts across the five tested microbiomes, it could result in significant alterations on a single microbiome basis. The example given by indomethacin indicated that the order Enterobacterales was enriched with increased chaperonin GroEL (HSP60 family) and co-chaperonin GroES (HSP10) (Fig. 6e), which have been implicated in infection and diseases pathology [51].

Our workflow still exhibits certain limitations. In particular, MS analysis is a time-consuming process. To this end, a fast-pass screening process should consider using techniques such as tandem mass tags (TMT) $[52,53]$ to multiplex multiple microbiome samples in one MS analysis. Furthermore, our workflow only measures the direct effects of compounds on the microbiome. In its current implementation, it does not take into account the host effect on the microbiome and/or the effects of drug metabolites produced by the host. Nevertheless, these metabolites could be tested along with the RapidAIM assay by additional analysis. Future efforts could be aimed at incorporating co-culture of host cells/tissue and gut bacteria [5456] into a high-throughput drug screening process for achieving more comprehensive insights on host-drugmicrobiome interaction. Metaproteomics is a tool that is orthogonal to other omics technologies [19]; hence, for the need of deeper investigations, RapidAIM could also be coupled with techniques such as metagenomics or metabolomics for a multiple dimension view of the microbiome interaction with drugs.

\section{Conclusion}

To date, the field of drug-microbiome interactions largely focuses on relative microbiome composition and microbial drug metabolism, with a limited understanding of the effects of pharmaceuticals on the absolute abundance and the function of the gut microbiome. A better understanding of these interactions is essential given that the drug effects on the microbiome biomass and 
functions may have important health consequences. Our workflow enabled the insights into both absolute abundances and functional responses of the gut microbiome to drugs using metaproteomics as the single analytical tool. We have shown that our workflow is robust, reproducible, and quantitative, and is easily adaptable for high-throughput drug screening applications.

\section{Methods}

\section{Stool sample preparation}

The Research Ethics Board protocol (\# 20160585-01H) for stool sample collection was approved by the Ottawa Health Science Network Research Ethics Board at the Ottawa Hospital. Stool samples were obtained from 5 healthy volunteers (age range 27-36 years; 3 males and 2 females). Exclusion criteria were as follows: IBS, IBD, or diabetes diagnosis; antibiotic use or gastroenteritis episode in the last 3 months; use of pro-/pre-biotic, laxative, or anti-diarrheal drugs in the last month; or pregnancy. All volunteers were provided with a stool collection kit, which included a 50-mL Falcon tube containing $15 \mathrm{~mL}$ of sterile phosphate-buffered saline (PBS) pre-reduced with $0.1 \%(\mathrm{w} / \mathrm{v})$ L-cysteine hydrochloride, a 2.5-mL-sterile sampling spoon (Bel-Art, USA), plastic wrap, gloves, and disposal bags. Briefly, each volunteer placed the plastic wrap over a toilet to prevent the stool from contacting water, collected $\sim 3 \mathrm{~g}$ of stool with the sampling spoon, and dropped the spoon into the prepared $50-\mathrm{mL}$ tube. The sample was immediately weighed by a researcher and transferred into an anaerobic workstation $\left(5 \% \mathrm{H}_{2}, 5 \% \mathrm{CO}_{2}\right.$, and $90 \% \mathrm{~N}_{2}$ at $\left.37{ }^{\circ} \mathrm{C}\right)$, where the tube was uncapped to remove $\mathrm{O}_{2}$ before homogenization with a vortex mixer. Then, the homogenate was filtered using sterile gauzes to remove large particles and obtain the fecal inoculum.

\section{Culturing of microbiota with drug treatments}

Each fecal inoculum was immediately inoculated at a concentration of $2 \%(\mathrm{w} / \mathrm{v})$ into a 96-well deep-well plate containing 1-mL culture medium and a compound dissolved in $5 \mu \mathrm{l}$ DMSO (or $5 \mu \mathrm{l}$ DMSO as the control) in each well. The culture medium contained $2.0 \mathrm{~g} \mathrm{~L}^{-1}$ peptone water, $2.0 \mathrm{~g} \mathrm{~L}^{-1}$ yeast extract, $0.5 \mathrm{~g} \mathrm{~L}^{-1} \mathrm{~L}$-cysteine hydrochloride, $2 \mathrm{~mL} \mathrm{~L}^{-1}$ Tween $80,5 \mathrm{mg} \mathrm{L}^{-1}$ hemin, 10 $\mu \mathrm{L} \mathrm{L}^{-1}$ vitamin $\mathrm{K} 1,1.0 \mathrm{~g} \mathrm{~L}^{-1} \mathrm{NaCl}, 0.4 \mathrm{~g} \mathrm{~L}^{-1} \mathrm{~K}_{2} \mathrm{HPO}_{4}$, $0.4 \mathrm{~g} \mathrm{~L}^{-1} \mathrm{KH}_{2} \mathrm{PO}_{4}, 0.1 \mathrm{~g} \mathrm{~L}^{-1} \mathrm{MgSO}_{4} \cdot 7 \mathrm{H}_{2} \mathrm{O}, 0.1 \mathrm{~g} \mathrm{~L}^{-1}$ $\mathrm{CaCl}_{2} \cdot 2 \mathrm{H}_{2} \mathrm{O}, 4.0 \mathrm{~g} \mathrm{~L}^{-1} \mathrm{NaHCO}_{3}, 4.0 \mathrm{~g} \mathrm{~L}^{-1}$ porcine gastric mucin (cat\# M1778, Sigma-Aldrich), $0.25 \mathrm{~g} \mathrm{~L}^{-1}$ sodium cholate, and $0.25 \mathrm{~g} \mathrm{~L}^{-1}$ sodium chenodeoxycholate [26]. The culture medium was sterile and had been prereduced overnight in an anaerobic workstation. Concentration of each compound was determined based on the assumption that maximal oral dosage of the drug distributed in $200 \mathrm{~g}$ average weight of the colon contents.
However, several compounds (i.e., cimetidine, ciprofloxacin, flucytosine, mesalamine, metformin, metronidazole, naproxen-sodium, paracetamol, rifaximin, sodium butyrate, and sulfasalazine) exceeded solubility in the given volume of DMSO $(5 \mu \mathrm{l})$. After confirming that these compounds still showed effect after a 10x dilution (as can be seen from hierarchical clustering in Supplementary Figure S3), the concentrations corresponding to the $1 / 10$ highest oral dosages were used for these compounds. Detailed catalog number and concentration of each compound is listed in Supplementary Table S1. After inoculation, the 96-well deep-well plate was covered with a sterile silicone gel mat with a vent hole for each well made by a sterile syringe needle. Then, the plate was shaken at $500 \mathrm{rpm}$ with a digital shaker (MS3, IKA, Germany) at $37{ }^{\circ} \mathrm{C}$ for $24 \mathrm{~h}$ in the anaerobic chamber.

\section{Metaproteomic sample processing and LC-MS/MS analysis} The sample processing was based on a previously reported metaproteomic sample processing workflow [57], we adapted it for 96-well plates (Supplementary Figure S1). Briefly, after culturing for $24 \mathrm{~h}$, each 96-well plate was transferred out of the anaerobic station and was immediately centrifuged at $300 \mathrm{~g}$ at $4{ }^{\circ} \mathrm{C}$ for $5 \mathrm{~min}$ to remove debris. With all plates sitting on ice, the supernatants were transferred into new 96-well deep-well plates for another two rounds of debris removal at $300 \mathrm{~g}$. The supernatants were then transferred to a new plate and centrifuged at $2272 g$ for $1 \mathrm{~h}$ to pellet the microbiota. The supernatant was removed, and the pelleted bacterial cells were washed three times with cold PBS in the same 96-well deep-well plate, pelleting the cells after each wash by a $2272 g$ centrifugation for $1 \mathrm{~h}$ at $4{ }^{\circ} \mathrm{C}$. The 96-well plate containing harvested bacterial cells was then stored overnight at $-80{ }^{\circ} \mathrm{C}$ before bacterial cell lysis and protein extraction. The lysis buffer was freshly prepared, containing $8 \mathrm{M}$ urea in 100 $\mathrm{mM}$ Tris-HCl buffer $(\mathrm{pH}=8.0)$, plus Roche PhosSTOP ${ }^{\mathrm{mm}}$ and Roche cOmplete ${ }^{\mathrm{Tm}}$ Mini tablets. Microbial cell pellets were then re-suspended in $150 \mu \mathrm{l}$ lysis buffer and lysed on ice using a sonicator (Q125 Qsonica, USA) with an 8-tiphorn probe. One hundred percent amplitude was used (i.e., 15.6 watts per sample), and four cycles of $30 \mathrm{~s}$ ultrasonication and $30 \mathrm{~s}$ cooling down were performed. Protein concentrations of the DMSO control samples were measured in triplicate using a detergent compatible (DC) assay (Bio-Rad, USA). Then, a volume equivalent to the average volume of $50 \mu \mathrm{g}$ of protein in the DMSO control samples was acquired from each sample and placed into a new 96well deep-well plate. The samples were reduced and alkylated with $10 \mathrm{mM}$ dithiothreitol (DTT) and $20 \mathrm{mM}$ iodoacetamide (IAA), followed by a $10 \times$ dilution using $100 \mathrm{mM}$ Tris- $\mathrm{HCl}(\mathrm{pH}=8.0)$ and tryptic digestion at $37^{\circ} \mathrm{C}$ for $18 \mathrm{~h}$ using $1 \mu \mathrm{g}$ of trypsin per well (Worthington Biochemical 
Corp., Lakewood, NJ). Digested peptides were desalted using a panel of lab-made 96-channel filter tips generated by inserting $9620 \mu \mathrm{l}$ filter tips into a 96-well cover mat and stacking each filter tip with $5 \mathrm{mg}$ of $10-\mu \mathrm{m} \mathrm{C} 18 \mathrm{col}-$ umn beads. After being washed twice with $0.1 \%$ formic acid $(\mathrm{v} / \mathrm{v})$, tryptic peptides were eluted with $80 \%$ acetonitrile $(\mathrm{v} / \mathrm{v}) / 0.1 \%$ formic acid $(\mathrm{v} / \mathrm{v})$.

After freeze-drying, each sample was re-dissolved in $100 \mu \mathrm{l} 0.1 \%$ formic acid $(\mathrm{v} / \mathrm{v})$, and $2 \mu \mathrm{l}$ of the solution (corresponding to $1 \mu \mathrm{g}$ of proteins in the DMSO control) was loaded for LC-MS/MS analysis in a randomized order. An Agilent 1100 Capillary LC system (Agilent Technologies, San Jose, CA) and a Q Exactive mass spectrometer (ThermoFisher Scientific Inc.) were used. Peptides were separated on a tip column $(75-\mu \mathrm{m}$ inner diameter $\times 50 \mathrm{~cm})$ packed with reverse phase beads $(1.9$ $\mu \mathrm{m} / 120 \AA$ ReproSil-Pur C18 resin, Dr. Maisch GmbH, Ammerbuch, Germany) using a 90-min gradient from 5 to $30 \%(\mathrm{v} / \mathrm{v})$ acetonitrile at a $200 \mathrm{~nL} / \mathrm{min}$ flow rate. $0.1 \%$ $(\mathrm{v} / \mathrm{v})$ formic acid in water was used as solvent $\mathrm{A}$, and $0.1 \% \mathrm{FA}$ in $80 \%$ acetonitrile was used as solvent $\mathrm{B}$. The MS scan was performed from 300 to $1800 \mathrm{~m} / \mathrm{z}$, followed by data-dependent MS/MS scan of the 12 most intense ions, a dynamic exclusion repeat count of two, and repeat exclusion duration of $30 \mathrm{~s}$ were used. The resolutions for MS and MS/MS were 70,000 and 17,500, respectively.

\section{Assessment of the equal-volume strategy}

Six dilutions of a single microbiome sample were prepared in triplicate wells and an equal volume was taken from each sample for tryptic digestion and LC-MS/MS analysis. Metaproteomic sample processing and analysis followed the same procedures stated above, and total peptide intensity was calculated. A DC protein concentration assay was also performed with each sample. Linearity between total protein concentration and total peptide intensity quantified by LC-MS/MS was then compared.

\section{Metaproteomics data analysis}

Protein/peptide identification and quantification, taxonomic assignment, and functional annotations were done using the MetaLab software (version 1.1.0) [27]. MetaLab is a software that automates an iterative database search strategy, i.e., MetaPro-IQ [29]. The search was based on a human gut microbial gene catalog containing 9,878,647 sequences from http://meta.genomics.cn/. In MetaLab, a spectral clustering strategy [27] was used for database construction from all raw files, then the peptide and protein lists were generated by applying strict filtering based on a FDR of 0.01 , and quantitative information for proteins was obtained with the maxLFQ algorithm on MaxQuant (version 1.5.3.30). Carbamidomethyl (C) was set as a fixed modification and oxidation (M) and N-terminal acetylation (Protein $\mathrm{N}$-term) were set as variable modifications. The matching between runs option was used. Instrument resolution was set as "High-High."

Total microbiome biomass was estimated for each sample by summing peptide intensities. Taxonomic identification was achieved by assigning peptide sequences to lineage of lowest common ancestor (LCA). The "peptide to taxonomy" database (pep2tax database) was selected for mapping identified peptides to the taxonomic lineages [27]. Bacteria, eukaryota, viruses, and archaea were included in the LCA calculation. Taxonomic biomass was quantified by summing the intensities of the peptides corresponding to each taxon. A Bray-Curtis dissimilarity-based approach [32] was applied for evaluating the variation of genus-level biomass contributions between drug-treated and DMSO control groups. Calculation of the Bray-Curtis distance was performed using the R package "vegan" [33].

The quantified protein groups were first filtered according to the criteria that the protein appears in $>80 \%$ of the microbiomes with at least one drug treatment. Then, LFQ protein group intensities of the filtered file were $\log _{2}$-transformed and normalized through quotient transformation $(x /$ mean) using the $\mathrm{R}$ package "clusterSim." Variance associated with the individual signature was evaluated by PerMANOVA test [31] based on BrayCurtis dissimilarities [32, 33] using the $\mathrm{R}$ package "vegan." Then, LFQ protein group intensities were processed by a ComBat process $[58,59]$ using iMetalab.ca [60] to remove possible batch effects between individual microbiomes. Using the ComBat-corrected data, an unsupervised non-linear dimensionality reduction algorithm, $\mathrm{t}$-distributed stochastic neighbor embedding ( $\mathrm{t}$ SNE) [34] was then applied to visualize similarities between samples using the $\mathrm{R}$ package "Rtsne." Parameter for the function Rtsne() were, perplexity $=10$, max_iter $=1200$ (number of iterations), other parameters were set as default. The $\mathrm{R}$ function geom_polygon implemented in ggplot2 was used to visualize the t-SNE results.

Functional annotations of protein groups, including COG and KEGG information, were obtained in the MetaLab software. In addition, KEGG ortholog (KO) annotation of protein FASTA sequences was conducted using GhostKOALA (https://www.kegg.jp/ghostkoala/) [61]. $\log _{2}$ fold-change of each drug-treated sample relative to the corresponding DMSO control was calculated using the abundances of proteins annotated to COG categories and COGs. Functional enrichment analysis was performed using the enrichment module on iMetalab.ca through inputting the list of COG functional proteins. Adjusted $p$ value cutoff was set at 0.05 for the enrichment analysis. We also visualized all functional responses (including COG, KEGG, NOG, and GO terms) using a $\mathrm{R}$ 
Shiny app, which is available at https://shiny.imetalab.ca/ RapidAIM_functional_response/. In this app, fold change of a function between treated and control microbiomes was visualized using boxplots, and statistical significances were calculated using Wilcoxon test.

\section{Statistical analysis}

We examined data distribution on all levels of data, and results indicated non-normal distributions of the dataset (examples shown in Supplementary Figures S12 and S13). Hence, a non-parametric statistical hypothesis test, the Wilcoxon rank sum test, was applied in statistical analyses. For multiple comparisons, $p$ values were adjusted using the Benjamini-Hochberg false discovery rate (FDR) procedure [62]. For multivariate analysis, partial leastsquares discriminant analyses (PLS-DA) based on ComBat-corrected protein group intensities were performed using MetaboAnalyst (http://www.metaboana lyst.ca/) [63]. PLS-DA model were evaluated by crossvalidation of $R^{2}$ and $Q^{2}$.

\section{Data visualizations}

Box plots, violin plots, hierarchical clustering, 3D scatter plots, heatmaps, PCA, and t-SNE were visualized using $\mathrm{R}$ packages ggplot2, gridExtra, scatterplot3d, and pheatmap. Pathway maps were visualized using iPATH 3 (https:// pathways.embl.de/) [64] and Pathview Web (https://pathview.uncc.edu/) [65]. Stacked column bars and functional enrichments were visualized on iMetaLab.ca.

\section{Supplementary information}

Supplementary information accompanies this paper at https://doi.org/10. 1186/s40168-020-00806-z

Additional file 1: Table S1. Information of 43 tested compounds.

Table S2. Significantly shifted species under drug treatment (one-sided Wilcoxon rank sum test, FDR-adjusted $p<0.05$ ).

Additional file 2: Figure S1. Establishment and step-by-step validation of the microplate-based metaproteomic sample preparation workflow of the RapidAIM assay. Figure S2. Assessment of the equal-volume digestion and LC-MS/MS analysis strategy. Figure S3. Data quality check of the POC dataset. Figure S4. Reproducibility of RapidAIM assay on different levels. Figure S5. Case study on microbiome $\mathrm{V}^{\prime}$ 's response to rifaximin. Figure $\mathbf{S 6}$. $\log _{2}$ fold-change of relative abundance at the genus level in response to each drug compared with the DMSO control. Figure S7. Score plots and cross-validations of seven PLS-DA models. Figure S8. $\log _{2}$ fold-change of functions at the COG protein level. Figure S9. String interaction of COG functional proteins significantly stimulated by diclofenac. Figure S10. Response of enzymes along the butyrate production from Acetyl-CoA. Figure S11. Phylum-specific functional responses to Berberine. Figure S12. Randomly selected LFQ intensities of protein groups showing heavy tailed distribution on the QQ plots. Figure S13. Randomly selected $\log _{2}$-fold changes of COGs showing heavy tailed distribution on the Q-Q plots.

\section{Abbreviations}

COG: Clusters of orthologous groups; DMSO: Dimethyl sulfoxide; FDR: Falsediscovery rate; FOS: Fructooligosaccharide; GABA: Gamma-aminobutyric acid; KEGG: Kyoto Encyclopedia of Genes and Genomes; LC-MS/MS: Liquid chromatography-tandem mass spectrometry; LFQ: Label-free quantification;
NSAIDs: Nonsteroidal anti-inflammatory drugs; PCA: Principle component analysis; PLS-DA: Partial least squares discriminant analysis; POC: Proof of concept; VIP: Variable importance in projection

\section{Acknowledgements}

The authors wish to thank Dr. Kendra Hodgkinson and Dr. Caitlin Simopoulos for editing the manuscript.

\section{Authors' contributions}

$D F, A S$, and $L L$ designed the study. JM coordinated the volunteers and collected the samples. $L L, X Z$, and $Z N$ developed the workflow and performed the experiments. $L \mathrm{~L}, \mathrm{ZN}, \mathrm{XZ}$, and $\mathrm{KC}$ analyzed and visualized the data. $L L, D F$, and $A S$ wrote the manuscript. All authors read and approved the final manuscript.

\section{Funding}

This work was supported by the Government of Canada through Genome Canada and the Ontario Genomics Institute (OGI-114 and OGl-149), CIHR grant (ECD-144627), the Natural Sciences and Engineering Research Council of Canada (NSERC, grant no. 210034), and the Ontario Ministry of Economic Development and Innovation (REG1-4450).

\section{Availability of data and materials}

All raw data from LC-MS/MS have been deposited to the ProteomeXchange Consortium (http://www.proteomexchange.org) via the PRIDE partner repository (dataset identifiers PXD012724 and PXD012725).

\section{Ethics approval and consent to participate}

The Research Ethics Board protocol (\# 20160585-01H) for stool sample collection was approved by the Ottawa Health Science Network Research Ethics Board at the Ottawa Hospital. All volunteers signed consent forms.

\section{Consent for publication}

Not applicable.

\section{Competing interests}

DF and AS have co-founded Biotagenics and MedBiome, clinical microbiomics companies. All other authors declare no competing interests.

\section{Author details}

${ }^{1}$ Department of Biochemistry, Microbiology and Immunology, Ottawa Institute of Systems Biology, Faculty of Medicine, University of Ottawa, Ottawa, Canada. ${ }^{2}$ Canadian Institute for Advanced Research, Toronto, Canada.

Received: 5 June 2019 Accepted: 12 February 2020

Published online: 11 March 2020

\section{References}

1. Zimmermann M, Zimmermann-Kogadeeva M, Wegmann R, Goodman AL. Separating host and microbiome contributions to drug pharmacokinetics and toxicity. Science. 2019;363:eaat9931.

2. Lynch SV, Pedersen $\mathrm{O}$. The human intestinal microbiome in health and disease. N Engl J Med. 2016;375:2369-79.

3. Jia W, Li H, Zhao L, Nicholson JK. Gut microbiota: a potential new territory for drug targeting. Nat Rev Drug Discov. 2008;7:123.

4. Maier L, Pruteanu M, Kuhn M, Zeller G, Telzerow A, Anderson EE, Brochado AR, Fernandez KC, Dose $H$, Mori $H$, et al. Extensive impact of non-antibiotic drugs on human gut bacteria. Nature. 2018:555:623-8.

5. Chen T, Li J, Chen T, Sun CC, Zheng Y. Tablets of multi-unit pellet system for controlled drug delivery. J Control Release. 2017;262:222-31.

6. Le Bastard Q, Al-Ghalith GA, Grégoire M, Chapelet G, Javaudin F, Dailly E, Batard E, Knights D, Montassier E. Systematic review: human gut dysbiosis induced by non-antibiotic prescription medications. Aliment Pharmacol Ther. 2017;47:332-45.

7. Urquhart L. FDA new drug approvals in Q2 2018. Nat Rev Drug Discov. 2018;17:536

8. Harvey AL, Edrada-Ebel R, Quinn RJ. The re-emergence of natural products for drug discovery in the genomics era. Nat Rev Drug Discov. 2015;14:111.

9. McDonald JAK, Schroeter K, Fuentes S, Heikamp-deJong I, Khursigara CM, de Vos WM, Allen-Vercoe E. Evaluation of microbial community 
reproducibility, stability and composition in a human distal gut chemostat model. J Microbiol Methods. 2013;95:167-74.

10. Van den Abbeele P, Belzer C, Goossens M, Kleerebezem M, De Vos WM, Thas O, De Weirdt R, Kerckhof F-M, Van de Wiele T. Butyrate-producing Clostridium cluster XIVa species specifically colonize mucins in an in vitro gut model. ISME J. 2012;7:949.

11. Van de Wiele T, Van den Abbeele P, Ossieur W, Possemiers S, Marzorati M. The Simulator of the Human Intestinal Microbial Ecosystem (SHIME ${ }^{\oplus}$ ). In: Verhoeckx K, Cotter P, López-Expósito I, Kleiveland C, Lea T, Mackie A, Requena T, Swiatecka D, Wichers H, editors. The Impact of Food Bioactives on Health: in vitro and ex vivo models. Cham: Springer International Publishing; 2015. p. 305-17.

12. Auchtung JM, Robinson CD, Britton RA. Cultivation of stable, reproducible microbial communities from different fecal donors using minibioreactor arrays (MBRAs). Microbiome. 2015;3:42.

13. Maier L, Pruteanu M, Kuhn M, Zeller G, Telzerow A, Anderson EE, Brochado AR, Fernandez KC, Dose $H$, Mori $H$, et al. Extensive impact of non-antibiotic drugs on human gut bacteria. Nature. 2018;555:623.

14. Props R, Kerckhof F-M, Rubbens P, De Vrieze J, Hernandez Sanabria E, Waegeman W, Monsieurs P, Hammes F, Boon N. Absolute quantification of microbial taxon abundances. ISME J. 2017;11:584-7.

15. Jian C, Luukkonen P, Yki-Järvinen H, Salonen A, Korpela K. Quantitative PCR provides a simple and accessible method for quantitative microbiome profiling. bioRxiv. 2018:478685.

16. Ranjan R, Rani A, Metwally A, McGee HS, Perkins DL. Analysis of the microbiome: advantages of whole genome shotgun versus $16 \mathrm{~S}$ amplicon sequencing. Biochem Biophys Res Commun. 2016;469:967-77.

17. Bashiardes S, Zilberman-Schapira G, Elinav E. Use of metatranscriptomics in microbiome research. Bioinform Biol Insights. 2016;10:19-25.

18. Milo R. What is the total number of protein molecules per cell volume? A call to rethink some published values. BioEssays. 2013;35:1050-5.

19. Mills RH, Vázquez-Baeza Y, Zhu Q, Jiang L, Gaffney J, Humphrey G, Smarr L, Knight R, Gonzalez DJ. Evaluating metagenomic prediction of the metaproteome in a 4.5-year study of a patient with Crohn's disease. mSystems. 2019;4:e00337-18.

20. Liu Y, Beyer A, Aebersold R. On the dependency of cellular protein levels on mRNA abundance. Cell. 2016;165:535-50.

21. Zhang X, Deeke SA, Ning Z, Starr AE, Butcher J, Li J, Mayne J, Cheng K, Liao $B, L i$, et al: Metaproteomics reveals associations between microbiome and intestinal extracellular vesicle proteins in pediatric inflammatory bowel disease. Nat Commun. 2018; 9:2873.

22. Zhang X, Chen W, Ning Z, Mayne J, Mack D, Stintzi A, Tian R, Figeys D. Deep metaproteomics approach for the study of human microbiomes. Anal Chem. 2017;89:9407-15.

23. Kleiner M. Metaproteomics: much more than measuring gene expression in microbial communities. mSystems. 2019;4:e00115-9.

24. Kleiner M, Thorson E, Sharp CE, Dong X, Liu D, Li C, Strous M. Assessing species biomass contributions in microbial communities via metaproteomics. Nat Commun. 2017:8:1558.

25. Zhang $X$, Figeys D. Perspective and guidelines for metaproteomics in microbiome studies. J Proteome Res. 2019.

26. Li L, Abou-Samra E, Ning Z, Zhang X, Mayne J, Wang J, Cheng K, Walker K, Stintzi A, Figeys D. An in vitro model maintaining taxon-specific functional activities of the gut microbiome. Nat Commun. 2019;10:4146.

27. Cheng K, Ning Z, Zhang X, Li L, Liao B, Mayne J, Stintzi A, Figeys D. MetaLab: an automated pipeline for metaproteomic data analysis. Microbiome. 2017:5:157.

28. Zhang X, Li L, Mayne J, Ning Z, Stintzi A, Figeys D. Assessing the impact of protein extraction methods for human gut metaproteomics. J Proteom. 2018;180:120-7

29. Zhang X, Ning Z, Mayne J, Moore Jl, Li J, Butcher J, Deeke SA, Chen R, Chiang C-K, Wen M, et al. MetaPro-IQ: a universal metaproteomic approach to studying human and mouse gut microbiota. Microbiome. 2016:4:31.

30. Brodribb AJ, Groves C. Effect of bran particle size on stool weight. Gut. 1978; 19:60.

31. Anderson MJ: Permutational multivariate analysis of variance (PERMANOVA). In Wiley StatsRef: Statistics Reference Online. Edited by Balakrishnan N, Colton T, Everitt B, Piegorsch W, Ruggeri F, Teugels JL; 2017: 1-15.

32. Consortium THMP, Huttenhower C, Gevers D, Knight R, Abubucker S, Badger JH, Chinwalla AT, Creasy HH, Earl AM, FitzGerald MG, et al. Structure, function and diversity of the healthy human microbiome. Nature. 2012;486:207.
33. vegan: Community ecology package. R package version 2.5-1. https://CRAN. R-project.org/package=vegan. Accessed 22 June 2018.

34. LJPvd M, Hinton GE. Visualizing high-dimensional data using t-SNE. J Mach Learn Res. 2008;9:2579-605.

35. Paulsen IT. Multidrug efflux pumps and resistance: regulation and evolution. Curr Opin Microbiol. 2003;6:446-51.

36. Onishi HR, Daoust DR, Zimmerman SB, Hendlin D, Stapley EO. Cefoxitin, a semisynthetic cephamycin antibiotic: resistance to beta-lactamase inactivation. Antimicrob Agents Chemother 1974;5:38.

37. Lu J, Vlamis-Gardikas A, Kandasamy K, Zhao R, Gustafsson TN, Engstrand L, Hoffner S, Engman L, Holmgren A. Inhibition of bacterial thioredoxin reductase: an antibiotic mechanism targeting bacteria lacking glutathione. FASEB J. 2012;27:1394-403.

38. Mayes PA, Bender DA. The pentose phosphate pathway \& other pathways of hexose metabolism. In: Murray RK, Granner DK, Mayes PA, Rodwell VW, editors. Harper's illustrated biochemistry. 26th ed. New York: Lange Medical Books/McGraw-Hill; 2003. p. 163-72.

39. Charteris WP, Kelly PM, Morelli L, Collins JK. Antibiotic susceptibility of potentially probiotic Bifidobacterium isolates from the human gastrointestinal tract. Lett Appl Microbiol. 1998;26:333-7.

40. Obad J, Šušković J, Kos B. Antimicrobial activity of ibuprofen: new perspectives on an "old" non-antibiotic drug. Eur J Pharm Sci. 2015;71:93-8.

41. Zhang $X$, Zhao $Y$, Zhang $M$, Pang $X$, Xu J, Kang C, Li M, Zhang C, Zhang Z, Zhang $Y$, et al. Structural changes of gut microbiota during berberinemediated prevention of obesity and insulin resistance in high-fat diet-fed rats. PLoS One. 2012;7:e42529.

42. Zhu L, Zhang D, Zhu H, Zhu J, Weng S, Dong L, Liu T, Hu Y, Shen X. Berberine treatment increases Akkermansia in the gut and improves high-fat dietinduced atherosclerosis in Apoe-/- mice. Atherosclerosis. 2018;268:117-26.

43. Cryan JF, Dinan TG. Mind-altering microorganisms: the impact of the gut microbiota on brain and behaviour. Nat Rev Neurosci. 2012;13:701.

44. Burokas A, Arboleya S, Moloney RD, Peterson VL, Murphy K, Clarke G, Stanton C, Dinan TG, Cryan JF. Targeting the microbiota-gut-brain axis: prebiotics have anxiolytic and antidepressant-like effects and reverse the impact of chronic stress in mice. Biol Psychiatry. 2017;82:472-87.

45. Kawaguchi M, Tashiro Y, Adachi T, Tamura Z. Changes in intestinal condition, fecal microflora and composition of rectal gas after administration of fructooligosaccharide and lactulose at different doses. Bifidobacteria and Microflora. 1993;12:57-67.

46. Swanson KS, Grieshop CM, Flickinger EA, Bauer LL, Chow J, Wolf BW, Garleb KA, Fahey JGC. Fructooligosaccharides and Lactobacillus acidophilus modify gut microbial populations, total tract nutrient digestibilities and fecal protein catabolite concentrations in healthy adult dogs. J Nutr. 2002;132:3721-31.

47. Lei XJ, Cheong JY, Park JH, Kim IH. Supplementation of protease, alone and in combination with fructooligosaccharide to low protein diet for finishing pigs. Animal Sci J. 2017;88:1987-93.

48. Albesa I, Becerra MC, Battán PC, Páez PL. Oxidative stress involved in the antibacterial action of different antibiotics. Biochem Biophys Res Commun. 2004;317:605-9.

49. Becerra MC, Albesa I. Oxidative stress induced by ciprofloxacin in Staphylococcus aureus. Biochem Biophys Res Commun. 2002;297:1003-7.

50. Becerra MC, Páez PL, Laróvere LE, Albesa I. Lipids and DNA oxidation in Staphylococcus aureus as a consequence of oxidative stress generated by ciprofloxacin. Mol Cell Biochem. 2006;285:29-34.

51. Ranford JC, Henderson B. Chaperonins in disease: mechanisms, models, and treatments. Mol Pathol. 2002:55:209-13.

52. Guirro M, Costa A, Gual-Grau A, Mayneris-Perxachs J, Torrell H, Herrero P, Canela N, Arola L. Multi-omics approach to elucidate the gut microbiota activity: metaproteomics and metagenomics connection. Electrophoresis. 2018;39:1692-701.

53. Thompson A, Schäfer J, Kuhn K, Kienle S, Schwarz J, Schmidt G, Neumann T, Hamon C. Tandem mass tags: a novel quantification strategy for comparative analysis of complex protein mixtures by MS/MS. Anal Chem. 2003;75:1895-904

54. Shah P, Fritz JV, Glaab E, Desai MS, Greenhalgh K, Frachet A, Niegowska M, Estes M, Jäger C, Seguin-Devaux C, et al. A microfluidics-based in vitro model of the gastrointestinal human-microbe interface. Nat Commun. 2016;7:11535.

55. Kim HJ, Lee J, Choi J-H, Bahinski A, Ingber DE. Co-culture of living microbiome with microengineered human intestinal villi in a gut-on-a-chip microfluidic device. J Vis Exp. 2016;54344. 
56. Jalili-Firoozinezhad S, Gazzaniga FS, Calamari EL, Camacho DM, Fadel CW, Bein A, Swenor B, Nestor B, Cronce MJ, Tovaglieri A, et al. A complex human gut microbiome cultured in an anaerobic intestine-on-a-chip. Nat Biomed Eng. 2019;3:520.

57. Mao L, Chen Q, Gong K, Xu X, Xie Y, Zhang W, Cao H, Hu T, Hong X, Zhan $Y$. Berberine decelerates glucose metabolism via suppression of mTORdependent HIF-1a protein synthesis in colon cancer cells. Oncol Rep. 2018 39:2436-42.

58. Chen C, Grennan K, Badner J, Zhang D, Gershon E, Jin L, Liu C. Removing batch effects in analysis of expression microarray data: an evaluation of six batch adjustment methods. PLoS One. 2011;6:e17238.

59. Nyamundanda G, Poudel P, Patil Y, Sadanandam A. A novel statistical method to diagnose, quantify and correct batch effects in genomic studies. Sci Rep. 2017;7:10849.

60. Liao B, Ning Z, Cheng K, Zhang X, Li L, Mayne J, Figeys D. iMetaLab 1.0: a web platform for metaproteomics data analysis. Bioinformatics. 2018:bty466-bty466.

61. Kanehisa M, Sato Y, Morishima K. BlastKOALA and GhostKOALA: KEGG tools for functional characterization of genome and metagenome sequences. J Mol Bio. 2016;428:726-31.

62. Benjamini Y, Hochberg Y. Controlling the false discovery rate: a practical and powerful approach to multiple testing. J Royal Stat Soc. 1995;57:289-300.

63. Chong J, Soufan O, Li C, Caraus I, Li S, Bourque G, Wishart DS, Xia J. MetaboAnalyst 4.0: towards more transparent and integrative metabolomics analysis. Nucleic Acids Res. 2018:46:W486-94.

64. Yamada T, Letunic I, Okuda S, Kanehisa M, Bork P. iPath2.0: interactive pathway explorer. Nucleic Acids Res. 2011;39:W412-5.

65. Luo W, Pant G, Bhavnasi YK, Blanchard JSG, Brouwer C. Pathview web: user friendly pathway visualization and data integration. Nucleic Acids Res. 2017; 45:W501-8.

\section{Publisher's Note}

Springer Nature remains neutral with regard to jurisdictional claims in published maps and institutional affiliations.

Ready to submit your research? Choose BMC and benefit from:

- fast, convenient online submission

- thorough peer review by experienced researchers in your field

- rapid publication on acceptance

- support for research data, including large and complex data types

- gold Open Access which fosters wider collaboration and increased citations

- maximum visibility for your research: over $100 \mathrm{M}$ website views per year

At $\mathrm{BMC}$, research is always in progress.

Learn more biomedcentral.com/submissions 